\title{
COMBINATORIAL EXAMPLES IN UNIVERSAL COALGEBRA
}

\author{
ROBERT C. DAVIS
}

\begin{abstract}
If an underlying-set functor different from the usual one is employed, the categories of multigraphs and of combinatorial systems become cotripleable over the category of sets. The category of multigraphs without loops and a common generalization are also examined.
\end{abstract}

1. Introduction. The fact that many classes of combinatorial structure are closed under disjoint union suggests that they might form categories that are cotripleable over the category of sets. However, the most obvious ways of trying to do this tend to lead to situations in which there is no right adjoint, or in which the dual Beck condition is not satisfied. It turns out that if part of the structure is included in the form of additional points adjoined to the underlying set, these difficulties may disappear.

2. Multigraphs. A multigraph $G$ consists of a set $V$ of vertices and a set $E$ of edges. We define the underlying set to be $U G=V \cup E$; that is, the underlying set contains one point for each vertex and one point for each edge, but forgets the incidence relations. A homomorphism is simply a function taking vertices to vertices, and edges to edges, and preserving incidence: if $e$ is one of the edges joining $v_{1}$ to $v_{2}$, then $f(e)$ is one of the edges joining $f\left(v_{1}\right)$ to $f\left(v_{2}\right)$. The multigraphs with these homomorphisms form a category $\mathcal{G}$.

THEOREM $1 . \mathcal{G}$ is cotripleable over the category of sets.

Proof. For each set $A$, we construct a multigraph $F(A)$ as follows. $A$ itself is the set of vertices. For $a, b$ in $A$, the edges joining $a$ and $b$ form a set in one-to-one correspondence with $A$. There is an obvious projection $p: U F(A) \rightarrow A$. Now suppose $G=(V, E)$ is any multigraph and $f: U G \rightarrow A$ any function. Then there is a unique homomorphism $g: G \rightarrow F(A)$ with $f=p U(G)$. In fact, for $v$ in $V, g(v)=f(v)$, while if $e$ in $E$ joins $v_{1}$ and $v_{2}$, then $g(e)$ is the edge labeled by $f(e)$ that joins $f\left(v_{1}\right)$ to $f\left(v_{2}\right)$. Thus, $F$ is the right adjoint to $U$.

For the dual of the Beck condition, suppose we have a diagram

$$
B \underset{z}{s} \underset{g}{\stackrel{f}{\leftrightarrows}} \underset{g}{\stackrel{t}{\rightrightarrows}} U H,
$$

Received by the editors November 29, 1982.

1980 Mathematics Subject Classification. Primary 18C15; Secondary 05C99, $08 \mathrm{C} 05$.

Key words and phrases. Cotripleable category, right adjoint, (dual) Beck conditions, multigraph, combinatorial system. 
where $f$ and $g$ are homomorphisms, $f z=g z, s z=1_{B}, t f=1_{U G}$, and $t g=z s$. We require that there be a unique multigraph structure on $B$ that makes $z=e q(f, g)$ into a homomorphism. This will be clear, provided that whenever $z(e)$ is an edge in $G$, the vertices $x, y$ joined by $z(e)$ are in the image of $z$. But $f(z(e))=g(z(e))$ is an edge in $H$ joining $f(x)$ to $f(y)$, and also joining $g(x)$ to $g(y)$. If $f(x)=g(x)$, then $x=t(f(x))=t(g(x))=z(s(x))$ and $y=z(s(y))$ are in the image of $z$. Otherwise, we must have $f(x)=g(y)$, and then likewise $x=z(s(y))$ and $y=z(s(x))$ are in the image of $z$.

3. Multigraphs without loops. These also form a cotripleable category. But it is not quite a subcategory of $\mathcal{G}$, since the definition of a homomorphism must be altered by the bizarre condition that if $e$ joins $v_{1}$ and $v_{2}$, and $f\left(v_{1}\right)=f\left(v_{2}\right)$, then $f(e)$ must be some vertex. The proof is almost identical, except that the loops in $F(A)$ are missing, and if $e$ joins $v_{1}$ to $v_{2}$, where $f\left(v_{1}\right)=f\left(v_{2}\right)$, then $g(e)$ is the vertex $f(e)$. The proof of the Beck condition is not altered since there $f$ must be one-to-one. In this category $U F(2)$ has 4 elements, so it seems to be a minimal example of a fundamental type of cotripleable category, playing a role similar to other examples such as $Z_{2}$-sets and sets with equivalence relations.

4. Combinatorial systems. A system [2] $S=(V . E)$ consists of a set $V$ of vertices, a set $E$ of blocks, and a function $\alpha: E \rightarrow P(V)$. As before, we define $U S=V \cup E$, with one point for each block, forgetting incidence, and homomorphisms take vertices to vertices, blocks and blocks, and preserve incidence $(\alpha f=f \alpha)$. Systems form a category $\delta$.

THEOREM 2. $\mathcal{S}$ is cotripleable over the category of sets.

Proof. For each set $A$, we construct a system $F(A)$ as follows. The set of vertices is $A$, and the set of blocks is $A \times P(A)$. Thus, the blocks are the subsets of $A$, but each one appears $|A|$ times, labeled by each element of $A$. The projection $p$ : $U F(A) \rightarrow A$ is obvious. If $f: U S \rightarrow A$, the homomorphism $g: S \rightarrow F(A)$ with $f=$ $p U(g)$ has $g(v)=f(v)$ on vertices, and if $e$ is a block of $S, g(e)$ is the subset $f(\alpha(e))$ (direct image) labeled by the point $f(e)$. Then $F$ is the right adjoint to $U$.

To consider the Beck condition, we may retain the notation of the proof of Theorem 1. Then what is needed is to see that when $z(e)$ is a block, all the elements of $\alpha(z(e))$ are in the image of $z$. If $x$ is in $\alpha(z(e))$ then $f(x)$ is in $f(\alpha(z(e)))=$ $\alpha(f(z(e)))=\alpha(g(z(e)))=g(\alpha(z(e)))$ so $f(x)=g(y)$ for some $y$ in $\alpha(z(e))$. Then $x=t(f(x))=t(g(y))=z(s(y))$ is in the image of $z$. From this it is easy to see that $B$ carries a unique system structure making $z$ into a homomorphism.

5. Generalized systems. Here we consider systems $S=(V, E)$ equipped with a function $\varphi: P(V) \rightarrow P(E)$, and $U S=V \cup E$. For multigraphs, with or without loops, and $B \subseteq V$, one can take $\varphi(B)$ to be the set of edges joining two vertices that are either both in $B$ or both not in $B$. For systems, one can take $\varphi(B)$ to be the set of blocks $e$ with $\alpha(e) \subseteq B$.

To see what homomorphisms should be, it is easiest to translate this description into the terms of the general characterization of quasicotripleable categories in [1]. A 
quasicotripleable category can be formed by sets $A$ equipped with an operation $\omega_{A}$ : $P(A) \rightarrow P(A)$, possibly subject to equational constraints, and homomorphisms $f$ : $A \rightarrow B$ satisfying $f^{-1}\left(\omega_{B}(X)\right)=\omega_{A}\left(f^{-1}(X)\right)$ for all $X \subseteq B$. This means that the colimit-preserving and dual Beck conditions are automatically met, though there may not necessarily be a right adjoint.

Now, given a system as described above, we can define for all $X \subseteq S=V \cup E$ the value of $\omega_{S}(X)$ to be $V \cup \varphi(V \cap X)$. All operations $\omega$ obtained by this means obey the equational scheme

$$
\omega\left(\bigcup_{\lambda}\left(B_{\lambda} \cap \omega\left(C_{\lambda}\right)\right) \cup \bigcup_{\mu}\left(D_{\mu} \cap\left(S-\omega\left(E_{\mu}\right)\right)\right)\right)=\omega\left(\bigcup_{\lambda} B_{\lambda}\right),
$$

and conversely if this condition is satisfied one may obtain a generalized system with $V=\bigcap\{\omega(X) \mid X \subseteq S\}$. Thus, the generalized systems form a quasicotripleable category $\mathscr{F}$.

THEOREM 3. F is cotripleable over the category of sets.

Proof. By the above remarks, we need only indicate the construction of the right adjoint. If $A$ is a set, $F(A)$ will have $V=A$ and $E=A \times P^{2}(A)$. The function $\varphi$ : $P(A) \rightarrow P(E)$ is defined by $\varphi(X)=A \times\left\{\mathcal{E} \in P^{2}(A) \mid X \in \mathcal{E}\right\}$ for all $X \subseteq A$. If $S$ is a system and $f: S \rightarrow A$, then the homomorphism $g$ is defined by $g(e)=(f(e), \alpha(e))$ for every block $e$ of $S$, where $\alpha(e)=\left\{X \subseteq A \mid e \in \varphi_{S}\left(f^{-1}(X)\right)\right\}$.

Every quasicotripleable category definable in terms of a single operation $\omega$ can be embedded in $\mathscr{F}$ as follows. If $A$ is an algebra in the category, one puts $A$ for both $V$ and $E$, and for $X \subseteq V, \varphi(X)=\omega_{A}(X) \subseteq E$. The action on a homomorphism is duplicated on the two components. Thus, $\mathscr{F}$ supplies the right adjoints missing in such familiar examples as sets equipped with an arbitrary relation, or topological spaces (where $\omega$ is closure and homomorphisms are continuous open maps). In this sense it serves as a universal quasicotripleable category.

\section{REFERENCES}

1. R. C. Davis, Quasicotripleable categories, Proc. Amer. Math. Soc. 35 (1972), 43-48.

2. J. E. Graver and M. E. Watkins, Combinatories with emphasis on the theory of graphs, Graduate Texts in Math., vol. 54, Springer-Verlag, New York, 1977.

Department of Mathematics, Southern Methodist University, Dallas, Texas 75275 